\title{
Excitons and Disorder in Molecular Nanotubes: A 2D Electronic Spectroscopy Study and First Comparison to a Microscopic Model
}

\author{
Jaroslaw Sperling, ${ }^{\dagger}$ Alexandra Nemeth,${ }^{\ddagger}$ Jürgen Hauer, ${ }^{\ddagger}$ Darius Abramavicius, ${ }^{\S}$ \\ Shaul Mukamel, ${ }^{\$}$ Harald F. Kauffmann," and Franz Milota ${ }^{*, \perp}$ \\ Newport Spectra-Physics, Guerickeweg 7, 64291 Darmstadt, Germany, Electronic Properties of Materials, \\ Faculty of Physics, University of Vienna, Strudlhofgasse 4, 1090 Vienna, Austria, Department of Chemistry, \\ 1102 Natural Sciences, University of California, Irvine, California 92697-2025, Ultrafast Dynamics Group, \\ Faculty of Physics, Vienna University of Technology, Wiedner Hauptstrasse 8 - 10, 1040 Vienna, Austria, and \\ Lehrstuhl für BioMolekulare Optik, Ludwig-Maximilians-Universität, Oettingenstraße 67, \\ 80538 München, Germany
}

Received: March 10, 2010; Revised Manuscript Received: June 14, 2010

\begin{abstract}
The efficiency of natural light-harvesting complexes relies on delocalization and directed transfer of excitation energy on spatially well-defined arrangements of molecular absorbers. Coherent excitation delocalization and long-range molecular order are also central prerequisites for engineering energy flows in bioinspired devices. Double-wall cylindrical aggregates have emerged as excellent candidates that meet these criteria. So far, the experimental signatures of exciton relaxation in these tubular supramolecules could not be linked to models encompassing their entire spatial structure. On the basis of the power of two-dimensional electronic spectroscopy, we characterize the motion of excitons in the three-fold band structure of the bitubular aggregate C8S3 through temporal, energetic, and spatial attributes. Accounting for intra- as well as interwall electronic interactions in the framework of a Frenkel exciton basis, we employ numerical computations using inhomogeneous and homogeneous microscopic models. The calculations on large but finite structures identify disorder-induced effects, which become increasingly relevant for higher energy states and give insight into the topology of the excited state manifold. Calculations in the infinite homogeneous limit capture the phenomena evidenced in the experimental two-dimensional patterns. Our results provide a basis for understanding recently reported correlated fluctuations of excitonic absorption bands and interband coherences in tubular aggregates.
\end{abstract}

\section{Introduction}

A novel ansatz in the development of artificial light harvesters is the transfer of the self-organization tendency of organic surfactants to molecular dyes. ${ }^{1}$ The approach relies on linking hydrophobic and hydrophilic substituents to aggregate forming molecular dyes with efficient excitonic couplings. Among such amphiphilic (hydrophilic and hydrophobic) chromophores, the class of cyanine derivatives has the outstanding ability to selforganize into tubular structures that result from the interplay between the hydrophobic effect and the dispersive interactions between $\pi$-electrons of the cyanine backbone. ${ }^{2}$ As illustrated in Figure 1a for a tubular aggregate formed by the dye $\mathrm{C} 8 \mathrm{~S} 3,{ }^{3}$ the spatial structure of the supramolecules can be well characterized on a mesoscopic scale. ${ }^{4}$ In a motif that is typical for molecular nanotubes, the $\mathrm{C} 8 \mathrm{~S} 3$ chromophores are wrapped into a double-wall cylinder, with an inner diameter of $\approx 10 \mathrm{~nm}$, a wall-to-wall distance of $\approx 4 \mathrm{~nm}$, and lengths approaching the micrometer scale. The complexes thereby strongly resemble the rod elements in light-harvesting chlorosomes of green bacteria, which contain a huge number of bacteriochlorophylls in cylindrical arrangements of comparable diameters and serve to supply the bacterial reaction center with excitation energy. ${ }^{5,6}$

* To whom correspondence should be addressed, E-mail: franz.milota@ univie.ac.at.

Newport Spectra-Physics.

* University of Vienna.

$\S$ University of California.

"Vienna University of Technology.

${ }^{\perp}$ Ludwig-Maximilians-Universität. a)

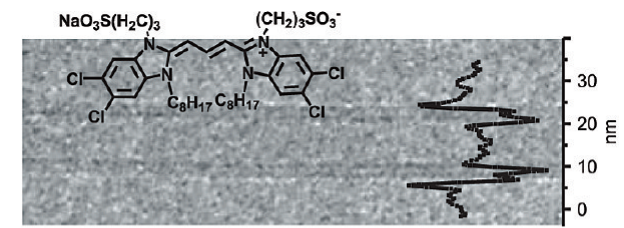

b)

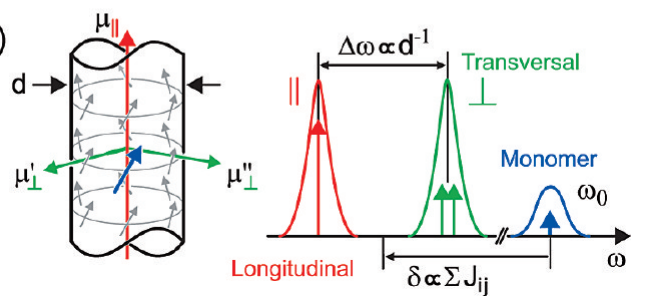

Figure 1. (a) Chemical structure of the monomer and a cryogenic transmission microscopy image of the aggregated sample C8S3. The curve on the right side shows the horizontally integrated image gray tone. (b) Schematics of the spatial arrangement of monomer transition moments in a single wall. Electronic coupling gives rise to one longitudinal $\left(\mu_{\|}\right)$and two (energy-degenerate) transversal transitions $\left(\mu_{\perp}^{\prime}, \mu_{\perp}^{\prime \prime}\right)$. The splitting $\Delta \omega$ between bands $\|$ and $\perp$ is inversely proportional to the diameter of the tubule. The spectral red-shift $\delta$ with respect to the monomer transition $\left(\omega_{0}\right)$ reflects interchromophore coupling strengths $\left(J_{i j}\right)$.

Even though the idea to use self-assembled tubules for building artificial light-harvesting complexes or to combine them as energy transport wires with photochemical reaction partners is tantalizing, up to now, experimental and theoretical studies addressing interwall exciton transport have not been clearly 
linked. As in linear aggregates, the spectroscopic properties (like, e.g., narrow linear absorption line-shapes) of cylindrical aggregates are determined by the close spatial proximity of coupled molecular transition dipoles and the consequent formation of Frenkel-excitons. ${ }^{7}$ However, in contrast to one-dimensional structures, the linear absorption (LA) of a periodic lattice with cylindrical symmetry splits into a longitudinal (coinciding with the cylinder axis) and two energy-degenerate transversal transitions (perpendicular to the cylinder axis, cf. Figure 1b). ${ }^{4,8}$ The experimental LA spectrum of the double-wall C8S3-aggregate, on the other hand, shows three bands, with two lower energy bands (band I/band II) polarized parallel and a third band (band III) polarized predominately transversal to the main tubular axis. The question arises to which extent this spectral shape can be regarded as an additive superposition of two single-wall contributions. In contrast to simple dimer systems, which can be easily classified into weak, intermediate, and strong coupling regimes by looking at the ratio of coupling strength to dephasing constant, ${ }^{9}$ this categorization is not that straightforward in large systems, where non-nearest neighbor couplings have to be considered.

There exist some discrepancies in the available literature dealing with the treatment of band-mixing effects due to interwall electronic couplings. In a combined experimental and theoretical analysis of C8S3, the interwall interactions have been assumed to be weak and were treated perturbatively. ${ }^{4}$ Recently, by looking into the slightly different derivative $\mathrm{C} 8 \mathrm{O} 3^{10}$ and employing systematic experimental two-dimensional electronic spectroscopy (2D-ES) studies, our group ${ }^{11,12}$ and Womick et al. ${ }^{13,14}$ identified off-diagonal peaks in 2D correlation spectra $\left(t_{2}=0\right)$, in favor of a nonperturbative nature of electronic interwall interactions. The cross-peaks in the correlation spectra map-out early electronic coherence and display excitonic tubeto-tube transfer dynamics in subsequent relaxation spectra as a quantum-dissipative process. ${ }^{15}$ Because these partly controversial results have been claimed for two morphologically slightly different derivatives, the extent of comparability is not clearly defined so far, as detailed experimental work on the bitubular system formed by $\mathrm{C} 8 \mathrm{~S} 3$ that might clarify the conflicting results is missing up-to now. The morphology of C8S3 is less complicated as compared to the supra-molecular structure formed by the dye C8O3. ${ }^{12}$ The supra-molecular helical structure found in $\mathrm{C} 8 \mathrm{O} 3$, which manifests itself by an additional electronic transition, is absent in the thio-analog C8S3. Thus, the coupling patterns including diagonal and off-diagonal peaks in 2D correlation and relaxation spectra can be expected to be less congested and more clearly arranged. This makes the simulation of the $2 \mathrm{D}$ response feasible on the basis of an atomistic model for the first time.

In this contribution, detailed 2D experiments on the threefold band structure of C8S3 aggregates are presented in conjunction with a novel theoretical-numerical simulation. A key point of the present work is to explore the nature of excitonic interwall couplings and wave function mixing that determine the particulars and details of the optical exciton dynamics in C8S3. We find evidence for strong electronic interwall interactions and their spectroscopic signatures from an atomistic computational analysis of 2D electronic correlation and relaxation spectra. The third-order nonlinear signals are reconstructed by a microscopic model employing a double-wall cylindrical structure. ${ }^{4}$ The experiments in combination with the microscopic cylindrical model simulations specifically trace tube-to-tube spatiotemporal excitonic transfer and are therefore a realistic extension of previous simulations performed on single-wall

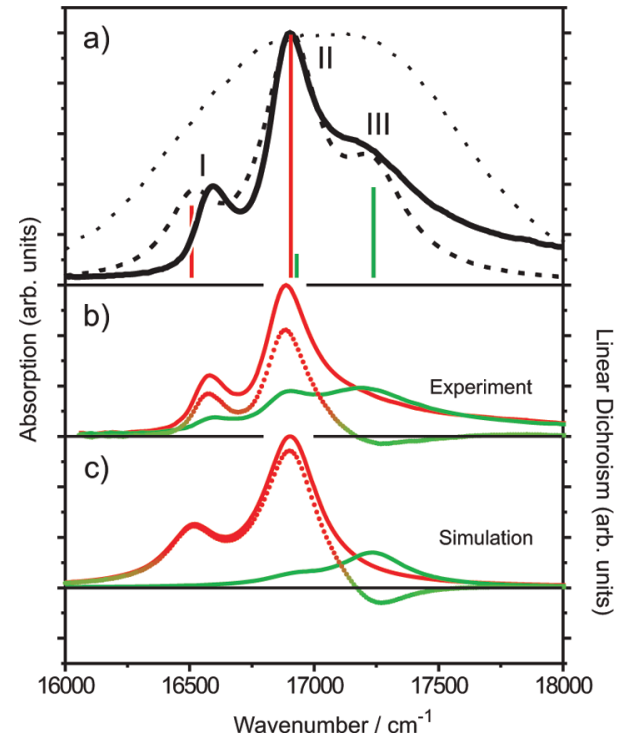

Figure 2. Linear absorption (LA) spectra from experiment and infinite model simulation. (a) Isotropic LA spectrum (solid line) with labeling of bands. The dotted line shows a typical laser pulse spectrum for comparison. The calculated isotropic LA is shown as a dashed line; vertical solid lines indicate the stick spectrum. (b) LA spectra under conditions of alignment in a flow-jet, recorded with light polarized parallel (red line, $\varepsilon_{\|}$) and vertical (green line, $\varepsilon_{\perp}$ ) to the flow direction. Colored dots show the corresponding linear dichroism (LD) spectrum $\left(\varepsilon_{\|}-\varepsilon_{\perp}\right)$. The corresponding simulated data is shown in (c).

cylinders and linear spectroscopic properties. ${ }^{8,16-18}$ In addition, the model is superior to the coupled multilevel energy scheme employed in previous works, ${ }^{11,12}$ which used experimental input quantities from 1D and 2D spectroscopy in the absence of structural information and energy-space relations.

The format of the paper is as follows. Section II is concerned with experimental details, describing the acquisition and evaluation of linear and nonlinear spectra. In Section III we bridge the gap between experiment and theory by presenting and discussing experimental and theoretical results on the tubular aggregate C8S3. Two approaches are presented; a finite (inhomogeneous) model with site disorder which is used to analyze linear spectra and an infinite (homogeneous) model without disorder that is compared to the 1D and 2D spectra. We close in Section IV with concluding remarks.

\section{Linear and Nonlinear Experiments}

Linear Spectra. For all experiments, C8S3 was dissolved in water and gently stirred for one week under exclusion of light. This stock solution $\left(c=5 \times 10^{-4} \mathrm{~mol} / \mathrm{L}\right)$ was diluted with water $(1: 1)$ before the measurements to yield an absorption of $\approx 0.4$ in the maximum. Due to the huge disparity in spatial dimensions, tubular aggregates tend to align along the flow direction in thin liquid films even at relatively low flow speeds. We take advantage of this fact by implementing a gravity-driven, wire-guided drop jet of approximately $200 \mu \mathrm{m}$ thickness, ${ }^{19}$ in which, based on comparison with calculations, we estimate an average angle of $20^{\circ}$ between the longitudinal tubular axes and the direction of the aligning flow. By switching the light polarization between parallel and vertical to the jet, this allows preferential excitation of either longitudinal or transversal transition moments (for which we use the terminology parallel and vertical, respectively, excitation in the following).

The isotropic LA spectrum of C8S3 in aqueous solution (cf. Figure 2) shows two well-resolved peaks of band I and II 
a)

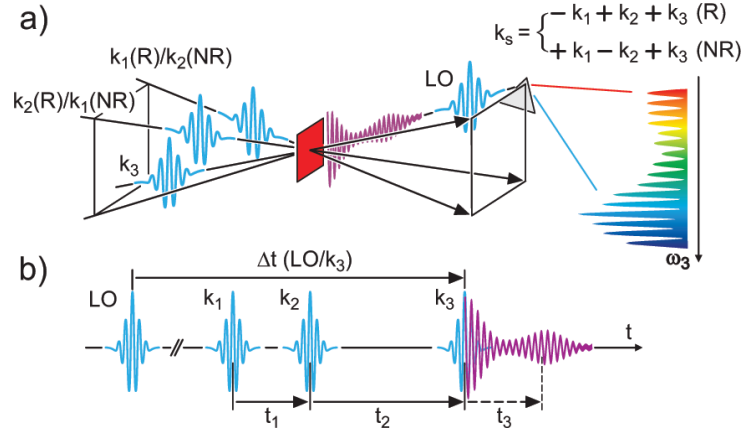

Figure 3. (a) Scheme of two-dimensional electronic spectroscopy. Depending on the timing sequence, three laser pulses (wavevectors $\mathbf{k}_{1}$, $\mathbf{k}_{2}, \mathbf{k}_{3}$ ) create a nonlinear signal in $\mathbf{k}_{\mathrm{s}}=-\mathbf{k}_{1}+\mathbf{k}_{2}+\mathbf{k}_{3}$ (rephasing $\mathrm{R}$ ) and $\mathbf{k}_{s}=+\mathbf{k}_{1}-\mathbf{k}_{2}+\mathbf{k}_{3}$ (nonrephasing $-\mathrm{NR}$ ), respectively. Spectral interference with a local oscillator pulse (LO) permits signal detection/ reconstruction in frequency domain $\left(\omega_{3}\right)$. (b) Definition of time-delays. After a stepwise recording of signals in a symmetric $t_{1}$-scan for a fixed value of $t_{2}$, a Fourier transform yields the $2 \mathrm{D}$ spectrum in $\left(\omega_{1}, \omega_{3}\right)$.

(located at $16590 \mathrm{~cm}^{-1}$ and $16900 \mathrm{~cm}^{-1}$ ) and a broad shoulder at higher energies (band III). As can be seen by comparison with the spectrum of our excitation pulses (cf. Figure 2), the spectral bandwidth allows to cover each of the three absorption bands with at least $80 \%$ of the maximal spectral pulse intensity.

Linear dichroism (LD) spectra were recorded with a homebuilt LD spectrometer, consisting of a halogen light source, collimating/focusing optics, a Glan-Thompson polarizer, the wire-guided jet, and a miniature CCD-spectrometer (USB2000, Ocean Optics). The LA substantially reshapes for parallel and vertical excitation conditions, as illustrated in Figure 2b. For parallel excitation, the two strong peaks of bands I and II dominate the spectrum $\left(\varepsilon_{\|}\right)$, and band III appears only as a weak spectral ridge. For vertical excitation $\left(\varepsilon_{\perp}\right)$ band III is the most intense feature, with a well-resolved peak maximum at 17185 $\mathrm{cm}^{-1}$. To considerable extent, this linear dichroism (LD $=\varepsilon_{\|}$ $-\varepsilon_{\perp}$, cf. Figure $2 b$ ) has been taken to support the notion of weak interwall interactions. Accordingly, the two main bands (bands I and II) have been assigned to longitudinal transitions of weakly interacting cylindrical monolayers, with transversal transitions that energetically coincide in a high energy wing (band III).

Two-Dimensional Electronic Spectroscopy. Two-dimensional electronic spectroscopy (2D-ES) correlates exciton frequencies that are traced during two time-intervals $\left(t_{1}, t_{3}\right)$ and allows testing against a particular scheme of electronic eigenstates by projecting coupled (isolated) absorbers into the offdiagonal (diagonal) part of a 2D correlation plot of conjugated frequencies $\omega_{1}$ and $\omega_{3}{ }^{20-22}$ Variation of the time-delay $\left(t_{2}\right)$ between the two observation windows visualizes exciton motion in a sequence of $2 \mathrm{D}$ relaxation spectra. ${ }^{11,23,24}$

The principle design of the experiment and the timing of the three excitation pulses $\left(\mathbf{k}_{1}, \mathbf{k}_{2}\right.$, and $\left.\mathbf{k}_{3}\right)$ are shown in Figure 3, panels $\mathrm{a}$ and $\mathrm{b}$, respectively. The spectra are acquired by scanning delay $t_{1}$ (separating the first two interactions of the sample with the pulse sequence) symmetrically around $t=0$, while keeping delay $t_{2}$ (between the second and third interaction) constant. For positive (negative) values of $t_{1}$, the scanning protocol collects rephasing (non-rephasing) contributions in the wavevector architecture $\mathbf{k}_{\mathrm{s}}=-\mathbf{k}_{1}+\mathbf{k}_{2}+\mathbf{k}_{3}\left(\mathbf{k}_{\mathrm{s}}=+\mathbf{k}_{1}-\mathbf{k}_{2}\right.$ $+\mathbf{k}_{3}$ ). At each delay, signal reconstruction (in $\omega_{3}$-domain) is achieved by spectral interferometry with a local oscillator pulse (cf. Figure 3).

As outlined in detail previously ${ }^{11,25} 2$ D-ES experiments are realized with an amplified titanium-sapphire (Ti:sa) laser system and a home-built noncollinear optical parametric amplifier (NOPA) ${ }^{26}$ that was tuned to $17300 \mathrm{~cm}^{-1}$ (spectral width 1250 $\mathrm{cm}^{-1}$ (fwhm), pulse duration $16 \mathrm{fs}$ ). To compensate the dispersive elements of the NOPA and the experimental setup, we apply a combination of Brewtser-angled chirped mirrors and a sequence of fused-silica prisms. ${ }^{27}$ For full characterization, effective compression of the pulses, and to ensure the shortest possible pulse duration at the location of the experiment, we apply zero-additional phase spectral interferometry for direct electric field reconstruction (ZAP-SPIDER). ${ }^{28}$

Upon the basis of the pioneering work of the groups of Miller ${ }^{29}$ and Fleming, ${ }^{30}$ we implement a diffractive optics based setup to realize heterodyne-detected two-dimensional electronic spectroscopy experiments. ${ }^{11}$ The NOPA output is split into two beams of equal intensity, one of which can be delayed with respect to the other (delay $t_{2}$ ), and focused onto a diffractive optical element to generate two phase-locked replicas of each of the two beams in the direction of the \pm first orders. All four pulses are collimated by a spherical mirror in such a way that each of them is centered at the corner of a square. Beam 2 passes a moveable glass-wedge pair to introduce time delay $t_{1}$ with a resolution of 5.3 as. Beams 1 and 3 pass identical static glasswedge pairs to balance the dispersion, whereas the LO is attenuated by a neutral density filter. All beams are focused toward the sample with another spherical mirror. The signal is generated in the same direction as the LO and is focused onto the slit of a thermo-electrically cooled CCD-spectrometer to record the spectral interferograms. To reduce unwanted higherorder effects and nonresonant signals from the solvent and to prevent the sample from too fast photodegradation the input beam is attenuated by a ND filter (after the NOPA) to yield not more than $0.5 \mathrm{~nJ}$ of energy in each of the excitation pulses. This energy corresponds to a fluence of $2.2 \times 10^{13}$ photons/ $\mathrm{cm}^{2}$ for each excitation pulse, which results in excitation of $0.08 \%$ of the molecules. For the present sample the scanning procedure for a given $t_{2}$-time requires stepping the $t_{1}$-delay from $-200 \mathrm{fs}$ to $+200 \mathrm{fs}$ in $0.65 \mathrm{fs}$ steps.

The evaluation of the interferograms follows the procedure outlined by Jonas ${ }^{20}$ and Brixner et al. ${ }^{30}$ Briefly, we extract the signal's spectral amplitude and phase by Fourier transformation, filtering, and back-transformation of every single interferogram. After division by the local oscillator spectrum, the data set is Fourier-transformed with respect to $t_{1}$. This yields a 2D spectrum for a given population time $t_{2}$, consisting of a real (absorptive) and imaginary (dispersive) part. The required absolute phase of the $2 \mathrm{D}$ complex signal is determined by projecting the real part onto spectrally resolved pump-probe spectra. ${ }^{20,30}$ Figure 4 shows the comparison of the spectrally resolved pump-probe data with the projections of the corresponding "phased" $2 \mathrm{D}$ spectra for 0 and $1000 \mathrm{fs}$ for vertical (left) and parallel (right) excitation.

\section{Connecting Experiment and Theory}

2D Correlation and Relaxation Patterns. Figure 5 shows the amplitude representations of the complex 2D signals $S^{(3)}\left(\omega_{1}, t_{2}, \omega_{3}\right)$ recorded for both parallel and vertical excitation. For a convenient visualization of electronic coupling patterns, the 2D amplitude representations are partly overlaid with the corresponding LA spectra. The latter are reflected in the signals along the diagonal $\left(\omega_{3}=\left|\omega_{1}\right|\right)$, which divides the plots into two triangular parts $\left(\omega_{3}<\left|\omega_{1}\right|\right.$ and $\left.\omega_{3}>\left|\omega_{1}\right|\right)$. Note however, that signals in 2D spectra scale differently with the transition dipole moment than those in LA spectra $\left(\left|\mu_{i}\right|^{4}\right.$ vs $\left.\left|\mu_{i}\right|^{2}\right)$. Additionally, the intensities of the 2D signals are reshaped by 


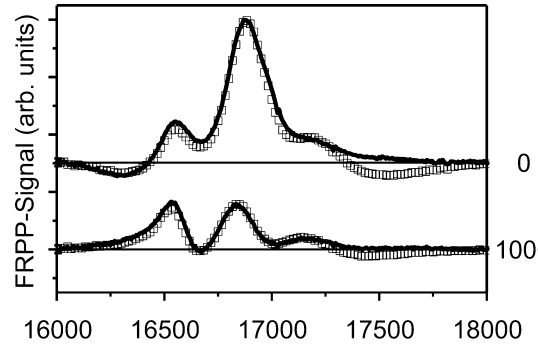

Wavenumber $/ \mathrm{cm}^{-1}$

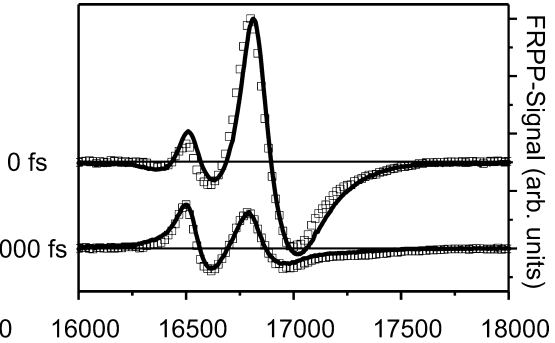

Wavenumber $/ \mathrm{cm}^{-1}$

Figure 4. Projections of the "phased" 2D spectra (solid line) for 0 and $1000 \mathrm{fs}$ in comparison with the spectrally resolved pump-probe data (white squares). Excitation vertical to the flow is depicted on the left, excitation parallel to the flow on the right side.
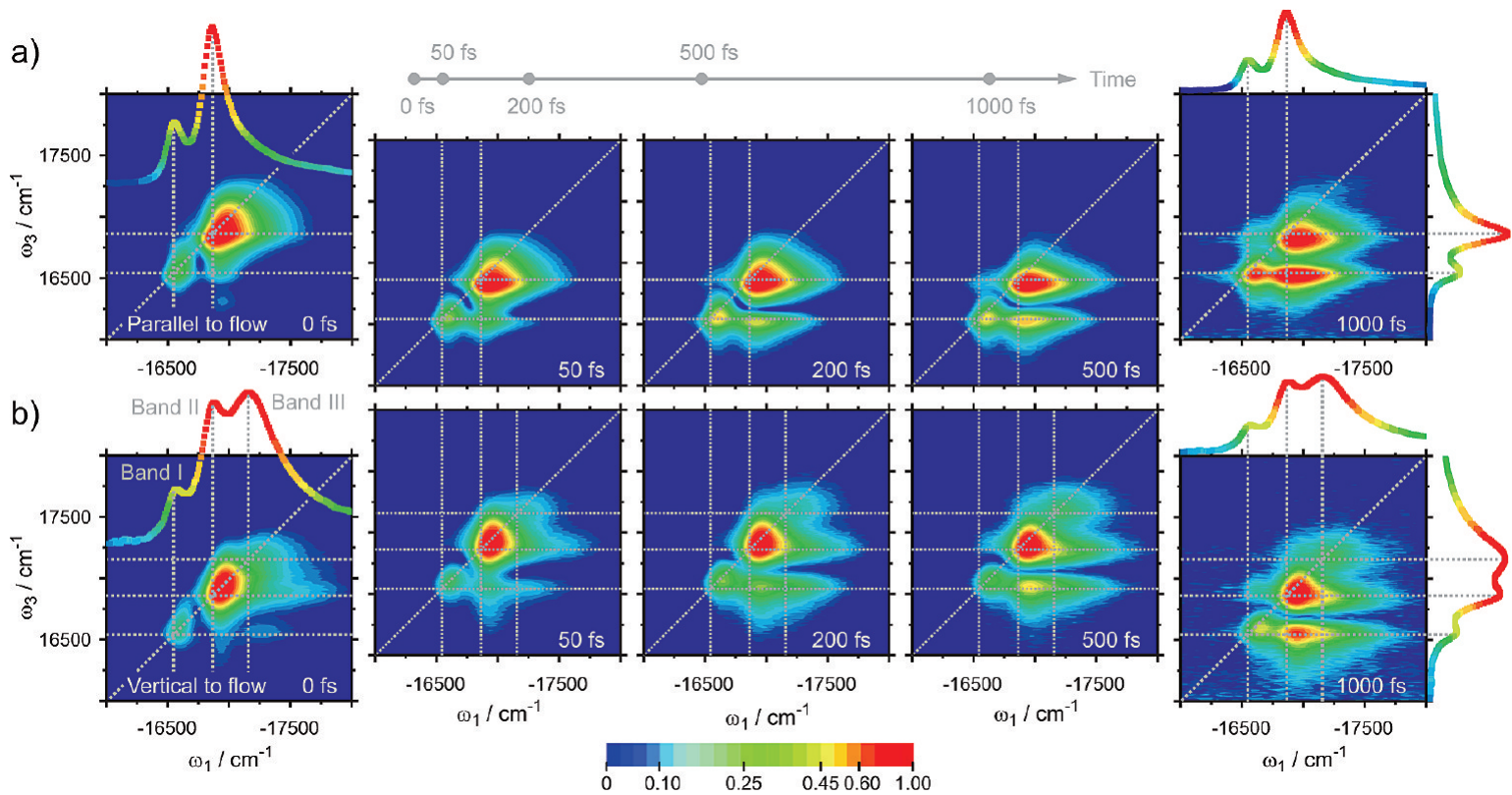

Figure 5. Amplitude representations of $2 \mathrm{D}$ electronic spectra recorded with excitation pulses polarized (a) parallel and (b) vertical to the flow direction (all spectra are normalized). From left to right, each row shows the $2 \mathrm{D}$ correlation spectrum $\left(t_{2}=0 \mathrm{fs}\right)$ and relaxation spectra recorded for $t_{2}$-delays of 50, 200,500, and $1000 \mathrm{fs}$, respectively. The corresponding (normalized) linear absorption spectra are shown for comparison in the first and last 2D spectrum of each sequence, along with dotted guidelines that mark the absorption maxima.

the finite pulse spectrum. The most striking 2D signals are the off-diagonal peaks being indubitable fingerprints of electronic couplings. ${ }^{11,12,23}$ They originate from typical combination transitions whose excitation dipoles are correlated by a common ground-state. ${ }^{31}$ Due to these off-diagonal cross-intensities, the correlation spectra $\left(t_{2}=0\right)$ in Figure 5 are asymmetric with respect to the diagonal.

For parallel excitation (Figure 5a), the two strong peaks I and II of the LA are recovered along the diagonal, and we observe around $20 \%$ of the maximal intensity at the band II/ band I off-diagonal coordinate in $\omega_{3}<\left|\omega_{1}\right|$. Peak III, which is not resolvable both in LA and as a diagonal peak in 2D-ES under parallel excitation, is directly detectable from the crosspeak signal between bands II and III whose intensity is proportional to $\left|\mu_{I I}\right|^{2} \cdot\left|\mu_{I I I}\right|^{2}$. Also for vertical excitation (Figure $5 b)$, all cross-intensities in $\omega_{3}<\left|\omega_{1}\right|$ are nonzero. The diagonal signal of band III is clearly seen in Figure 5b, although its intensity is substantially weaker than one would expect from its corresponding peak intensity in LA. Unlike for parallel excitation in Figure 5a, a cross-peak between bands III and I is resolved without difficulty under perpendicular polarization. Generally, off-diagonal intensities reveal electronic couplings between all of the three bands in the aggregate, the variation in the coupling patterns reflecting the dominant orientations of transition dipole moments. Parallel excitation intensifies the cross-intensity between the predominantly longitudinal transitions of bands I and II. Vertical excitation increases the relative contribution of band III and highlights its correlation with both of the two lower energy bands.

Apart from the differences in the correlation plots $\left(t_{2}=0\right)$ for parallel and vertical excitation, the temporal evolution of the relaxation spectra $\left(t_{2}=50,200,500\right.$, and $\left.1000 \mathrm{fs}\right)$ is governed by comparable tendencies. A striking feature is the growing-in of an intense cross-peak $\left(\omega_{3}<\left|\omega_{1}\right|\right)$, that arises via exciton relaxation from band II into band I (cf. Figure 5). At increasing $t_{2}$-delays, the off-diagonal signals grow in intensity and acquire a more and more elliptic shape with the long axis parallel to the $\omega_{1}$-axis, thereby extending from the $\left|\omega_{1}\right|-$ coordinate of band II far into higher frequencies. Also the diagonal contours of band I and band II become pronouncedly horizontally streaked. Because of simultaneous energy uphill transfer at longer waiting times (i.e., on a slower time scale), a much weaker off-diagonal feature appears at reversed coordinates $\left(\omega_{3}>\left|\omega_{1}\right|\right)$. At the same time, the relative intensity of band II decreases, whereas the relative diagonal peak intensity of band I steadily increases. This is an unbiased observation and a convincing proof of concurrent intertube exciton transfer driven by electronic coupling and relaxation, thereby conveniently illustrating the gain in information content by spreading the system's response into two dimensions. 

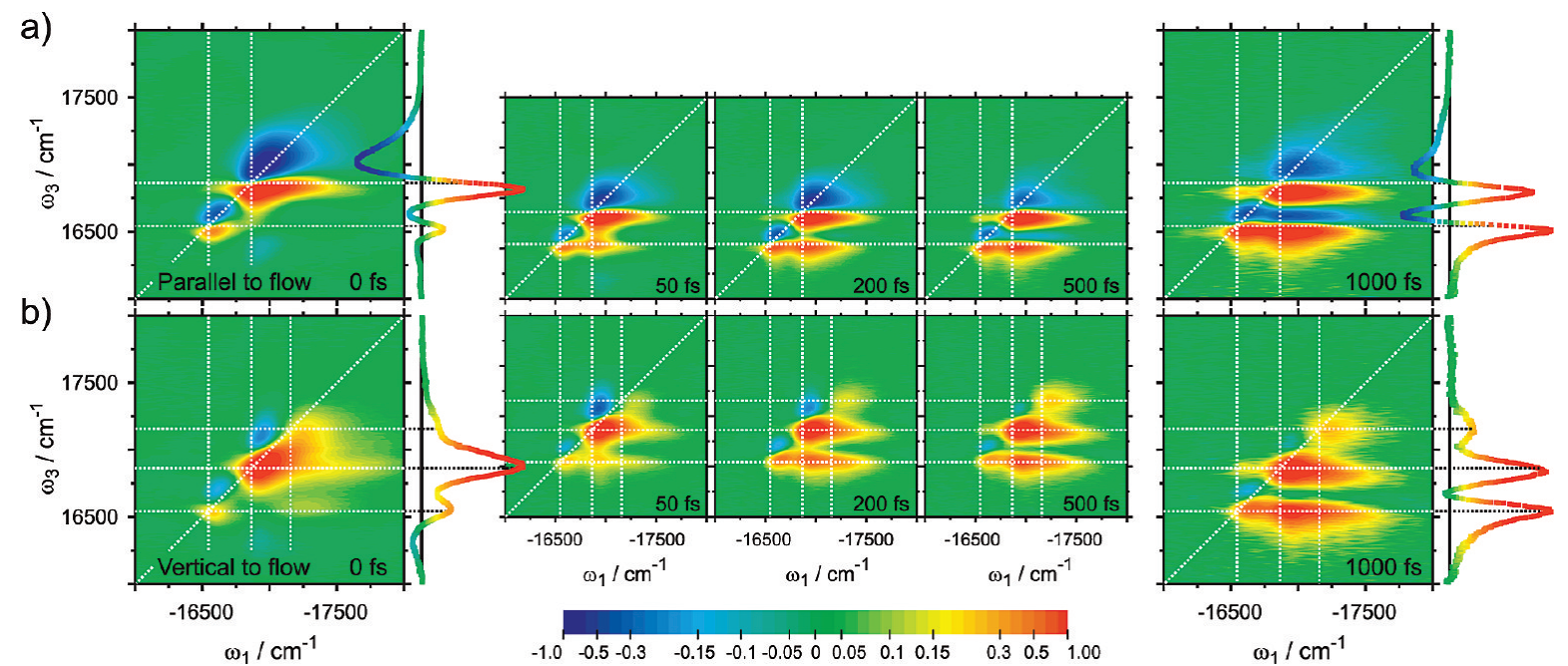

Figure 6. Absorptive parts of $2 \mathrm{D}$ electronic spectra recorded with excitation pulses polarized (a) parallel and (b) vertical to the flow direction. Same delay times as in Figure 5. The curves in the side panels of the first and the last column show the 2D signals integrated along $\omega_{1}$, and are equivalent to frequency resolved pump-probe spectra. All spectra are normalized to their maximum absolute value.

Depending on whether the system evolves in a one-exciton coherence or a coherence between a one- and a two-exciton state during $t_{3}$, absorptive 2D-ES signal parts of coupled absorbers feature both positive and negative contributions..$^{21,30,32}$ In the language of pump-probe spectroscopy, 2D peaks with negative sign are related to excited-state absorption (ESA) from the one- into the two-exciton band, whereas positive features can be assigned to ground state bleaching (GSB) and stimulated emission (SE) ${ }^{21,22}$ Since transitions between successively higher exciton manifolds are blue-shifted with respect to each other, ESA contributions to the 2D signal become shifted off of the diagonal into $\omega_{3}>\left|\omega_{1}\right|$. Turning to the $2 \mathrm{D}$ absorptive correlation spectra shown in Figure 6, we observe this effect for both lower energy bands I and II. In particular for parallel excitation, ESA signals are strong and contribute significantly to all of the recorded relaxation spectra (cf. Figure 6a). For vertical excitation, the negative signals are generally weaker, and essentially disappear within $500 \mathrm{fs}$ (cf. Figure 6b). The transition probabilities into the two-exciton manifold are enhanced for oneexciton states with longitudinal transition dipoles.

Beyond these aspects, the sequences of 2D absorptive spectra resemble the evolution of diagonal and off-diagonal intensities in the amplitude plots. Although correlation spectra $\left(t_{2}=0\right)$ are dominated by the intense signal of band II, the relaxation spectra highlight the evolution of the off-diagonal peak in $\omega_{3}<$ $\left|\omega_{1}\right|$ (simultaneously gaining in intensity and ellipticity) and the appearance of a considerably weaker cross-peak in $\omega_{3}>\left|\omega_{1}\right|$. Within $1 \mathrm{ps}$, the motion of relaxing excitons thus reshapes the diagonal signals of band I and band II into peaks of almost equal intensity.

Our experimental findings prompt us to draw the following conclusions. First, 2D electronic spectra prove that all of the excitonic bands of $\mathrm{C} 8 \mathrm{~S} 3$ are coupled and share a common ground state. Second, intensity growth of cross-peaks with increasing waiting time $t_{2}$ images ongoing exciton population relaxation. Third, since a cross-peak imaging exciton transfer in a $2 \mathrm{D}$ relaxation spectrum is proportional to the product of the squared transition dipole magnitudes of both of the states involved $\left(\propto\left|\mu_{i}\right|^{2}\left|\mu_{j}\right|^{2}\right)$, it permits observing the process as long as at least one of the states absorbs strong enough. In our case, the formation of streaked profiles, despite the only weak diagonal intensities at high frequencies, reflects the character- istics of excitonic states to substantially change across the spectrum of eigenstates. We substantiate these statements in the following.

Construction of a Microscopic Model. As the 2D experiments (cf. Figure 5 and Figure 6) demonstrate, essential aspects of the relaxation dynamics in tubular aggregates may be presumed to originate from site disorder and the consequent variation of the spatio-energetic exciton characteristics across the spectrum. In contrast to the assessable number of coupled pigments in most of the natural antennas currently studied in nonlinear experiments, ${ }^{33-35}$ molecular nanotubes are huge with respect to their building-blocks. The sheer number of molecules building up the tubular structure is an obstacle in any theoretical approach that aims to account for individual site-properties, like site-orientation or static site-disorder. In C8S3, roughly one hundred sites fill a tubular volume of one nanometer length, 2,4 so that roughly $10^{4}$ sites would be needed to set up a reasonably converged structure with a length to diameter ratio of approximately 10:1. An atomistic calculation of the nonlinear response of $\mathrm{N}$ excitonically coupled sites requires explicit information of the $N$ single- and the $N(N-1) / 2$ double-excited eigenstates. ${ }^{36}$ Even though we clearly perceive disorder effects from the streaking of the diagonal and off-diagonal peaks in the experimental two-dimensional electronic spectra, a simulation of nonlinear spectra within a microscopic model that includes disorder effects is too expensive. Therefore, in order to account for disorder effects on the one hand and nevertheless keep the computational expenses manageable on the other hand, we apply a two-fold strategy. In a first step, to account for the experimental disorder evidenced by the streaks in the $2 \mathrm{D}$ electronic spectra, we analyze the manifold of excitonic states beyond the homogeneous limit, by introducing energetic and spatial site disorder in a finite model structure and restrict ourselves to the calculation of linear signals (LA, LD). In a second step, for simulating the third-order nonlinear signals at reasonable computational costs, we employ an infinite model in the homogeneous limit.

In the present work, for the double-walled cylindrical model diameters of 10 and $3.3 \mathrm{~nm}$ are assumed for the outer and inner rings, respectively. The two rings are equidistantly occupied with 24 (outer wall) and 16 (inner wall) sites, reflecting the occupation ratio of roughly 60 versus 40 sites as deduced from 


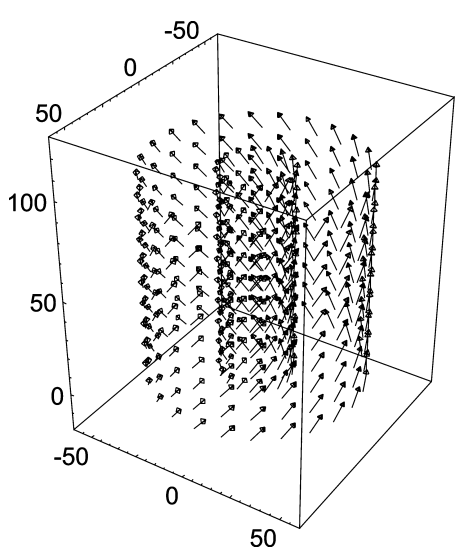

Figure 7. Lattice used for simulations. Tick labels indicate the spatial dimensions in angstroms. The clip shows a stack of 10 adjacent unit cells, arrows indicate the orientation of the molecular transition dipole moments (scaled for a correspondence of $1 \mathrm{D}$ to $1 \AA$ ).

experiment. ${ }^{4,18}$ Since we observe the key spectral properties to remain essentially unaltered for a broad range of tubular diameters if we keep the interwall distance fixed (if site-perring occupation numbers are appropriately adjusted), this approach allows to reduce the number of chromophores. All other microscopic parameters compare well to previous estimates. ${ }^{4}$ We use a lattice constant (distance of adjacent rings along the longitudinal cylinder axis) of $1.1 \mathrm{~nm}$, the site transition energies are set to $\omega_{0}=18380 \mathrm{~cm}^{-1}$, and site-to-site couplings are calculated within the extended dipole model, assuming two charges $Q= \pm 0.41 e$ ( $e$, electron charge) separated by $l=0.56$ $\mathrm{nm}$ (corresponding to a molecular transition dipole moment of $\mu=11.03 \mathrm{D}$ ). In an iterative procedure (carried out in the homogeneous limit, see below), we initialize the above parameters, restrict transition dipole orientations to tangential planes, and perform a fit to the experimental line-shapes under variation of the dipole alignments. Doing so, we obtain the transition dipoles of the inner and outer wall oriented at an angle of 32 and $38^{\circ}$, respectively, to the longitudinal cylinder axis (cf. Figure 7).

We stress that in comparison to the brick-layer lattice model used in previous studies, ${ }^{4,18}$ the model employed in this work is less sophisticated. The brick-layer lattice model relies on wrapping a $2 \mathrm{D}$ lattice structure with $2 \mathrm{D}$ bricks representing the individual molecular sites onto a cylindrical surface along a wrapping vector. Since the wrapping vector has to commensurate with the lattice periodicity but is otherwise arbitrary, such an approach has more freedom in its fitting parameters and allows the inclusion of, for example, helical ring stacking in a straightforward manner. Within the approach taken here, some aspects related to the detailed spatial orientation might be missed. However, we emphasize that in the present work, we aim at a first comparison with nonlinear spectral data, keeping an eye on a simultaneous description of the essential signatures in linear and nonlinear experiments. A comparison of experimental and theoretical results strongly supports the notion that the essential physical aspects are captured accordingly. The fact that we are able to reasonably reproduce linear as well as circular dichroism properties justifies this approximation.

Disorder and Spatio-Energetic Exciton Properties. The finite model calculations are performed on a structure containing 80 adjacent double-rings (i.e., a total of 3200 sites), building up a tubule of roughly $90 \mathrm{~nm}$ length. To model orientational disorder, we allow for a random deviation of site-transition moments from their ideal lattice position by at most $10 \%$. For energetic disorder, site transition energies are randomly assigned from a Gaussian distribution with a width $\sigma_{\omega}=225 \mathrm{~cm}^{-1}$ centered at $\omega_{0}=18380 \mathrm{~cm}^{-1}$. These parameters for static disorder ensure exciton properties to be converged for the chosen size of the system (i.e., to be independent of a further increase of the tubular length). Dynamical disorder is modeled by coupling every site to a bath mode (an overdamped Brownian oscillator with a reorganization energy of $100 \mathrm{~cm}^{-1}$ and a correlation time of $50 \mathrm{fs}) .^{22}$ By calculating the electronic siteto-site couplings, the exciton wave functions can be obtained by diagonalization of the Frenkel-Exciton Hamiltonian. The averaged $\left(10^{4}\right.$ realizations $)$ linear spectra are calculated employing the cumulant expansion of Gaussian fluctuations (CGF) approach. $^{37}$

Figure 8 illustrates the essential characteristics of the excited state manifold. The LA and LD spectra of the model structure(s) are shown in Figure 8a, where the inset demonstrates the convergence of the LA spectra with increasing cylinder lengths. As expected from theoretical considerations, each of the two cylinders features one longitudinal transition at lower energies and one (degenerate) transversal transition at higher energies, with the energetic separation between these two being inversely proportional to the tube diameter (cf. also Figure 1b). Hence, considering both cylinders in the double-wall architecture in the absence of interwall interactions, the energetic separation between the two transitions is intrinsically decreased for an isolated outer wall. Apart from this notion, the number of states effectively contributing to the absorption spectrum is diminished for the outer wall, so that absorption results from a lower number of states, which in turn leads to a narrowing of the absorption line shape. On the other hand, the absolute absorption strength of the outer wall is higher than that of the inner wall, due to the larger number of sites in the former. Upon calculating the absorption spectrum under inclusion of interwall interactions, a redistribution of oscillator strengths is observed (cf. the rightmost panel in Figure 8a). On the lower energy side, the spectra show two longitudinal transitions, closely resembling the original longitudinal transitions of the isolated walls. However, the energetically higher lying transversal transitions deviate strongly when comparing the linear combination of the inner and outer wall with the coupled double-wall system. These transitions are broadly scattered energetically, with low oscillator-strengths of the individual states only. This is the case even for very low values of disorder. Upon inclusion of realistic values of disorder and system-bath interaction strengths of the individual sites, we thus obtain a three-band structure in linear absorption. Note that the finite model calculations with low disorder (cf. insets in Figure 8a) eventually converge into the infinite (homogeneous) limit results discussed below.

In Figure 8b, we plot inverse participation ratios (excitation delocalization lengths) against absorption frequencies for the inner wall, outer wall, and the double wall structure for a single (randomly chosen) realization of disorder (statistical averaging does not affect our conclusions). Each dot represents an excitonic state and is colored according to the (normalized) absolute value of the corresponding transition dipole moment $\left(\mu_{i}\right)$. A comparison of the rightmost panel in Figure 8 with the linear absorption spectrum of C8S3 allows to classify bands I-III according to the underlying transitions. For each finite tubular segment, there are only a few dominant states that contribute to band I, which can be characterized to be of highly localized nature. For band II, it is still a relatively small number of states that determine the absorption peak. However, their delocalization lengths span a wide range of values, precluding a simple characterization of the absorption band. Finally, band 

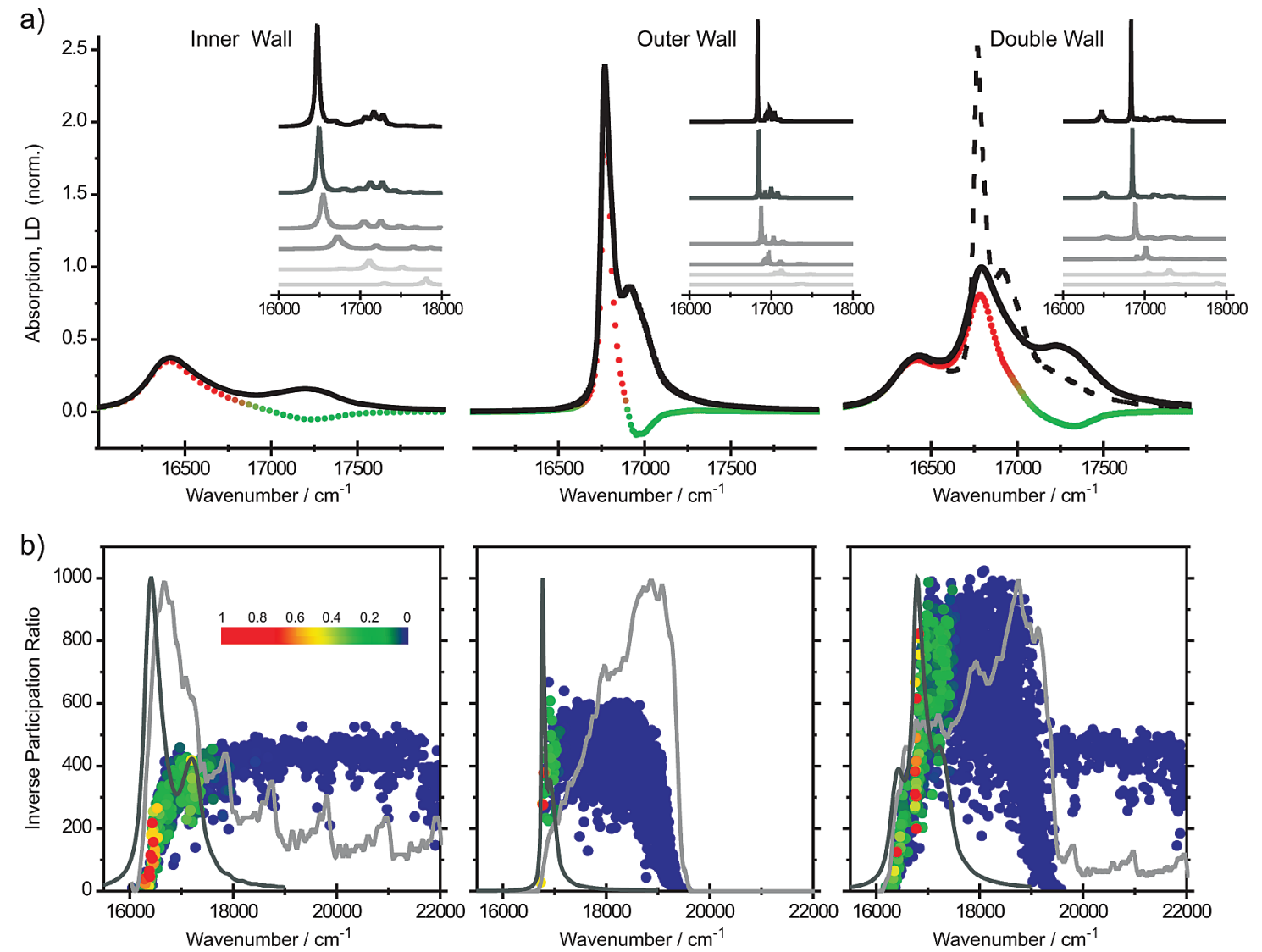

Figure 8. Characteristics of the one-exciton manifold from finite model calculations including disorder. (a) From left to right: averaged absorption spectrum of an isolated inner wall, an isolated outer wall, and the double-wall structure (all spectra are normalized to the double-wall LA). Colored dots show the corresponding LD. In the rightmost spectrum (double-wall), the sum of the inner and outer wall LA is shown for comparison (dashed line). The inset shows the convergence of the spectra (calculated at low values of disorder) as the number of rings is increased from 5 to 10 , 20 , 40, 60, and 80 rings (bottom to top, same absolute scale). (b) Inverse participation ratio versus exciton energy for inner wall (left), outer wall (middle), and double wall (right). All plots are for a single (randomly chosen) realization of disorder. Each dot represents an excitonic state, its color indicating the normalized transition dipole moment. The plotting sequence of points thereby follows increasing transition strengths, ensuring that a particular state can be overlaid only by a state with stronger absorption. The density of states (gray line) and the absorption spectrum (dark gray line) are shown as overlay.

III originates from a large number of only weakly absorbing excitonic states, which are less densely spaced in energy and are strongly delocalized.

For extracting the predominant spatial location of the excitonic wave functions in terms of the inner or outer tubular wall, we show in Figure 9, for the same data set, the sums of the wave function amplitudes collected on sites of the outer wall only. Recalling that for every exciton wave function a summation of amplitudes over all sites gives unity, this fraction tends to zero for excitons that mainly reside on the inner wall, while approaching unity if the wave functions are spread on the outer wall only. The functional form of this plot, which resembles a rectangular shape with a rounded edge on its low energy side, is revealing. Although the localization of excitons within band I, in fact, takes place on the inner wall, the states within band II are not only dispersed in delocalization lengths, but are also highly scattered in their spatial properties with respect to the double-wall structure. The effect is less pronounced for band III excitons, which can be crudely described as excitations that are equally shared between both of the two aggregate walls. Again, we point out that the strong interwall delocalization of all but the lowest energy states falsifies a straightforward classification of the aggregate's absorption bands.

Modeling Spectral Properties in the Homogeneous Limit. To reconstruct the linear as well as nonlinear signals of C8S3 within a microscopic (homogeneous) model, we employ a

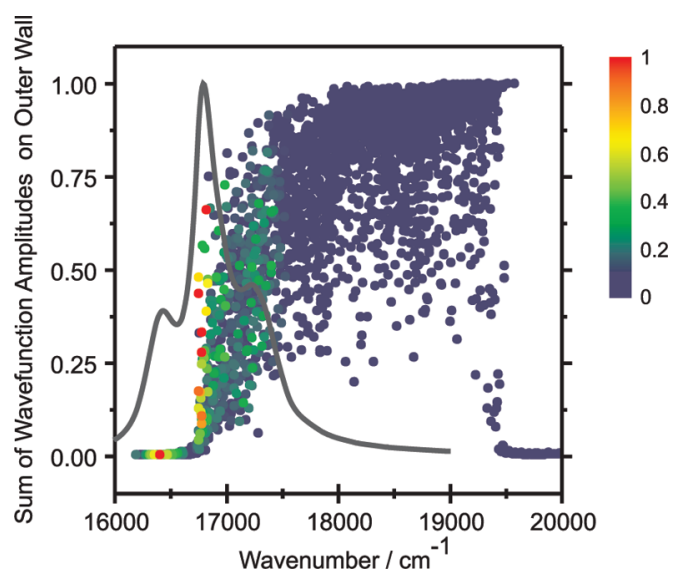

Figure 9. Sum of wave function amplitudes located on the outer wall for a single realization of disorder in the finite model calculations. Each dot represents an excitonic state, its color indicating the normalized transition dipole moment. The plotting sequence of points thereby follows increasing transition strengths, ensuring that a particular state can be overlaid only by a state with stronger absorption. Dark gray line shows the averaged LA spectrum.

1D periodic lattice (structural parameters as described above), whose unit cell contains two circular arrangements of transition dipoles. In the following, we label the sites inside a single unit cell by indices $m$ and identify each cell by its 
position vector $\mathbf{R}$. Each chromophore is treated as an identical two-level system. Due to translational invariance, the chromophore coupling $J_{m m^{\prime}}\left(\mathbf{R}-\mathbf{R}^{\prime}\right)$ is a function of the distance between the cells $\mathbf{R}$ and $\mathbf{R}^{\prime}$. The real-space Hamiltonian is: ${ }^{38}$

$$
\hat{H}=\sum_{\mathbf{R R}^{\prime}} \sum_{m n} J_{m n}\left(\mathbf{R}-\mathbf{R}^{\prime}\right) \hat{B}_{\mathbf{R} m}^{\dagger} \hat{B}_{\mathbf{R}^{\prime} n}+\frac{\Delta}{2} \sum_{\mathbf{R}} \sum_{m} \hat{B}_{\mathbf{R} m}^{\dagger 2} \hat{B}_{\mathbf{R} m}^{2}
$$

where $\hat{B}_{\mathbf{R} m}^{\dagger}$ is the exciton creation operator on the $m$ th chromophore in the $\mathbf{R}$ th conjugate annihilation operator. We use Boson statistics for the excitons with commutation relations $\left[\hat{B}_{\mathbf{R} m}, \hat{B}_{\mathbf{R}^{\prime} n}^{+}\right]=\delta_{m n} \delta_{\mathbf{R}^{\prime}}$. This model is called softcore boson model. The first term in the Hamiltonian represents one-exciton site energies $\varepsilon=J_{m m}(0)$ and resonant interactions, whereas the second term is a double-exciton binding parameter: taking $\Delta \rightarrow \infty$ we recover the two-level chromophore model.

The one-exciton states of this system are the Bloch states with wave functions

$$
\Psi_{\mathbf{R} m}^{\mathbf{q} \lambda}=\frac{1}{\sqrt{L}} \mathrm{e}^{-i \mathbf{q R}} \phi_{m \lambda}(\mathbf{q})
$$

where $L$ is the number of unit cells. Each eigenstate has a pair of quantum numbers $\mathbf{q} \lambda$, where $\lambda$ denotes different Davydov's subbands in the one-exciton band, and $\mathbf{q}=(\pi / L)[-1,1]$, with a step $\delta \mathbf{q}=(2 \pi / L)$, is the momentum. $\phi$ are the one-exciton states of a unit cell:

$$
\sum_{n} J_{m n}(\mathbf{q}) \phi_{n \lambda}(\mathbf{q})=\varepsilon_{\lambda}(\mathbf{q}) \phi_{m \lambda}(\mathbf{q})
$$

where

$$
J_{m n}(\mathbf{q})=\sum_{\mathbf{R}} \mathrm{e}^{i \mathbf{q} \mathbf{R}} J_{m n}(\mathbf{R})
$$

and $\varepsilon_{\lambda}(\mathbf{q})$ is the exciton energy at $\lambda$ Davydov's sub-band.

Exciton response to the optical fields is given in terms of many-exciton propagators - the exciton Green's functions. The single-exciton Green's function is $G_{\mathbf{q} \lambda}(t)=\theta(t) \exp \left[-i \varepsilon_{\lambda}(\mathbf{q}) t-\right.$ $\left.\gamma_{\lambda}(\mathbf{q}) t\right]$, where $\gamma_{\lambda}(\mathbf{q})$ is the exciton dephasing. The linear response function essential for calculating the LA and LD spectra is given by ${ }^{37}$

$$
R^{(1)}=\sum_{\lambda}\left|\mu_{\lambda}\right|^{2} G_{\lambda}(t)
$$

where $\mu_{\lambda}=L_{m} \mu_{m} \phi_{m \lambda}$ is the single-exciton transition dipole (the summation is over a single-cell). Note that we assumed $L a \ll$ $\bar{\lambda}$, where $a$ is the lattice constant, and $\bar{\lambda}$ is the optical wavelength, thus only zero-momentum exciton states contribute to the response; thus, the $\mathbf{q}=0$ parameter can be neglected.

The third-order response also depends on the double-exciton states. Using the quasi-particle representation, the third-order response can be calculated from the Green's function solution of the Nonlinear Exciton Equations (NEE).${ }^{38,39}$ For the photonecho phase-matching direction, $\mathbf{k}_{\mathrm{s}}=-\mathbf{k}_{1}+\mathbf{k}_{2}+\mathbf{k}_{3}$, we find

$$
\begin{aligned}
& R^{(3)}\left(t_{3}, t_{2}, t_{1}\right)=\sum_{\lambda_{4} \lambda_{3} \lambda_{2} \lambda_{1}} \sum_{\lambda_{2}^{\prime \prime} \lambda_{3} \lambda_{2}^{\prime} \lambda_{1}^{\prime}} \mu_{\lambda_{4}} \mu_{\lambda_{3}} \mu_{\lambda_{2}} \mu_{\lambda_{1}} \times \\
& \int_{0}^{\infty} \mathrm{d} \tau G_{\lambda_{4}}\left(t_{3}-\tau\right) V_{\lambda_{4} \lambda_{1}^{\prime} \lambda_{2}^{\prime} \lambda_{3}^{\prime}} G_{\lambda_{1}^{\prime}}^{*}(\tau) G_{\lambda_{2}^{\prime} \lambda_{3}^{\prime} \lambda_{2}^{\prime \prime} \lambda_{3}}^{(Y)}(\tau) G_{\lambda_{2}^{\prime \prime} \lambda_{1}^{\prime}, \lambda_{2} \lambda_{1}}^{(N)}\left(t_{2}\right) G_{\lambda_{1}}^{*}\left(t_{1}\right)
\end{aligned}
$$

Here, $V$ is the "scattering potential" obtained by transforming the second term in the Hamiltonian to the single-exciton eigenstate basis, $G^{(Y)}$ is the double-exciton Green's function, and $G^{(N)}$ is the single-exciton density-matrix Green's function; both in the single-exciton basis. This expression reflects the interaction and propagation sequence in third-order response, that is, the first interaction generates one exciton that propagates according to $G^{*}$, then the second interaction generates a density matrix in single-exciton space that propagates according to $G^{(N)}$. After the third interaction we have factorized the propagation into $G_{\lambda_{1}^{\prime}}^{*}$ and $G_{\lambda^{\prime} \lambda_{3}^{\prime}, \lambda_{2}^{\prime \prime} \lambda_{3}}^{(Y)}$ to account for double-exciton states. These particles finally interact through the scattering potential and generate the signal at $\lambda_{4}$. Similar to the linear response, we included only the zero-momentum states that do interact with the field.

To avoid the explicit calculation of $G^{(Y)}$, which involves finding all double-exciton states, we describe their resonances using the exciton scattering matrix $\Gamma_{\lambda_{4} \lambda_{3} \lambda_{2} \lambda_{1}}$. In the frequency domain we have

$$
G^{(Y)}(\omega)=G^{(0)}(\omega)+G^{(0)}(\omega) \Gamma(\omega) G^{(0)}(\omega)
$$

where $G_{\lambda_{4} \lambda_{3}, \lambda_{2} \lambda_{1}}^{(0)}(\omega)=\delta_{\lambda_{4} \lambda_{2}} \delta_{\lambda_{3} \lambda_{1}} I_{\lambda_{2} \lambda_{1}}(\omega)$ is the free-double-exciton Green's function (when excitons do not interact) with $I_{\lambda_{2} \lambda_{1}}(\omega)$ $=\left(\omega-\varepsilon_{\lambda_{2}}-\varepsilon_{\lambda_{1}}+i \gamma_{\lambda_{2}}+i \gamma_{\lambda_{1}}\right)^{-1}$.

The response function using this scattering matrix after applying double Fourier transformation is

$$
\begin{aligned}
& R^{(3)}\left(\Omega_{3}, t_{2}, \Omega_{1}\right)= \\
& \sum_{\lambda_{4} \lambda_{3} \lambda_{2} \lambda_{1}} \sum_{\lambda_{2}^{\prime} \lambda_{1}^{\prime}} \mu_{\lambda_{4}} \mu_{\lambda_{3}} \mu_{\lambda_{2}} \mu_{\lambda_{1}} \times G_{\lambda_{4}}\left(\Omega_{3}\right) \Gamma_{\lambda_{4} \lambda_{1}^{\prime} \lambda_{2}^{\prime} \lambda_{3}}\left(\Omega_{3}+\varepsilon_{\lambda_{1}^{\prime}}+\right. \\
& \left.\quad i \gamma_{\lambda_{1}^{\prime}}\right) I_{\lambda_{2}^{\prime} \lambda_{3}}\left(\Omega_{3}+\varepsilon_{\lambda_{1}^{\prime}}+i \gamma_{\lambda_{1}^{\prime}}\right) G_{\lambda_{2}^{\prime} \lambda_{1}^{\prime}, \lambda_{2} \lambda_{1}}^{(N)}\left(t_{2}\right) G_{\lambda_{1}}^{*}\left(\Omega_{1}\right)
\end{aligned}
$$

The scattering matrix is calculated using the Dyson equation, and its final form for two-level molecules is:

$$
\Gamma_{\lambda_{4} \lambda_{3} \lambda_{2} \lambda_{1}}(\omega)=\sum_{m n} \phi_{m \lambda_{4}} \phi_{m \lambda_{3}} \phi_{n \lambda_{2}} \phi_{n \lambda_{1}}\left[D^{-1}(\omega)\right]_{m n}
$$

and

$$
\begin{aligned}
& D_{m n}(\omega)= \\
& \quad \frac{1}{L} \sum_{\mathbf{q}} \sum_{\lambda_{2} \lambda_{1}} \frac{\phi_{m \lambda_{2}}(\mathbf{q}) \phi_{m \lambda_{1}}(-\mathbf{q}) \phi_{n \lambda_{2}}^{*}(-\mathbf{q}) \phi_{n \lambda_{1}}^{*}(\mathbf{q})}{\omega-\varepsilon_{\lambda_{2}}(\mathbf{q})-\varepsilon_{\lambda_{1}}(-\mathbf{q})+i \gamma_{\lambda_{2}}(\mathbf{q})+i \gamma_{\lambda_{1}}(-\mathbf{q})}
\end{aligned}
$$

The exciton density matrix Green's function is obtained from the Redfield equation for the density matrix: ${ }^{22}$

$$
\frac{\mathrm{d}}{\mathrm{d} t} \rho_{\lambda \lambda^{\prime}}=-i\left(\varepsilon_{\lambda}-\varepsilon_{\lambda^{\prime}}\right) \rho_{\lambda \lambda^{\prime}}-\sum_{\lambda^{\prime \prime} \lambda^{\prime \prime \prime}} K_{\lambda \lambda^{\prime}, \lambda^{\prime \prime} \lambda^{\prime \prime \prime}} \rho_{\lambda^{\prime \prime} \lambda^{\prime \prime \prime}}
$$


where $K$ is the Redfield relaxation rate matrix. We use the secular approximation for the density matrix, so that the populations (diagonal elements in the density matrix) and the coherences (off diagonal elements in the scattering matrix) evolve independently. The populations follow the Pauli master equation, and the coherences show exponentially damped oscillations. The Redfield rate matrix then attains the form:

$$
K_{\lambda \lambda^{\prime}, \lambda^{\prime \prime} \lambda^{\prime \prime \prime}}=\delta_{\lambda \lambda^{\prime}} \delta_{\lambda^{\prime \prime} \lambda^{\prime \prime \prime}} K_{\lambda \lambda, \lambda^{\prime \prime} \lambda^{\prime \prime}}+\delta_{\lambda \lambda^{\prime \prime}} \delta_{\lambda^{\prime} \lambda^{\prime \prime \prime}}\left(1-\delta_{\lambda \lambda^{\prime}}\right) \gamma_{\lambda \lambda^{\prime}}^{(N)}
$$

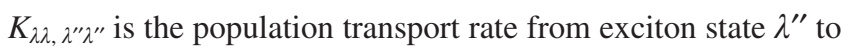
$\lambda$, and $\gamma_{\lambda \lambda}^{(N)}$ is the dephasing rate for interband coherence (i.e., coherence between single exciton states).

The final signal is obtained by convoluting the response function with the envelopes of the optical fields (semi-impulsive limit). The signal expressions become very simple if pulse overlapping regions can be neglected. We then have

$$
\begin{gathered}
S^{(3)}\left(\Omega_{3}, t_{2}, \Omega_{1}\right)=\sum_{\lambda_{4} \lambda_{3} \lambda_{2} \lambda_{1}} \sum_{\lambda_{2}^{\prime} \lambda_{1}^{\prime}} \mu_{\lambda_{4}} \mu_{\lambda_{3}} \mu_{\lambda_{2}} \mu_{\lambda_{1}} \times \\
E_{4}^{*}\left(\varepsilon_{\lambda_{4}}-\omega_{4}\right) E_{3}\left(\omega_{3}-\varepsilon_{\lambda_{3}}\right) E_{2}\left(\omega_{2}-\varepsilon_{\lambda_{2}}\right) E_{1}^{*}\left(\varepsilon_{\lambda_{1}}-\omega_{1}\right) \times \\
G_{\lambda_{4}}\left(\Omega_{3}\right) \Gamma_{\lambda_{4} \lambda_{1}^{\prime} \lambda_{2}^{\prime} \lambda_{3}}\left(\Omega_{3}+\varepsilon_{\lambda_{1}^{\prime}}+i \gamma_{\lambda_{1}^{\prime}}\right) I_{\lambda_{2}^{\prime} \lambda_{3}}\left(\Omega_{3}+\varepsilon_{\lambda_{1}^{\prime}}+\right. \\
\left.i \gamma_{\lambda_{1}^{\prime}}\right) G_{\lambda_{2}^{\prime} \lambda_{1}^{\prime}, \lambda_{2} \lambda_{1}}^{(N)}\left(t_{2}\right) G_{\lambda_{1}}^{*}\left(\Omega_{1}\right)
\end{gathered}
$$

Calculation of the signal requires knowledge of the relaxation parameters. These are calculated assuming that each chromophore is coupled to a statistically independent bath represented by a single overdamped Brownian oscillator. It is characterized by a spectral density of transition energy fluctuations, $\tilde{\varepsilon}(t):^{40}$

$$
C^{\prime \prime}(\omega)=\frac{1}{2} \int \mathrm{d} t \mathrm{e}^{i \omega \tau}\langle[\tilde{\varepsilon}(t), \tilde{\varepsilon}(0)]\rangle=2 l \frac{\omega \Lambda}{\omega^{2}+\Lambda^{2}}
$$

The relaxation rates in real space can then be calculated using the second-order perturbation theory in fluctuations and taking the Markovian (fast fluctuation) limit. For our system, using the auxiliary function ${ }^{22}$

$$
\begin{aligned}
M^{( \pm)}(\omega)= & \int_{0}^{\infty} \mathrm{d} t \mathrm{e}^{i \omega t} \times \\
& \int \frac{\mathrm{d} \omega}{2 \pi} C^{\prime \prime}(\omega)\left[\operatorname{coth}\left(\frac{\beta \hbar \omega}{2}\right) \cos (\omega t) \mp i \sin (\omega t)\right]
\end{aligned}
$$

we obtain

$$
\begin{gathered}
\gamma_{\lambda}=\sum_{\lambda^{\prime}} M^{(+)}\left(\varepsilon_{\lambda}-\varepsilon_{\lambda^{\prime}}\right) \phi_{\lambda \lambda^{\prime}} \\
K_{\lambda^{\prime} \lambda^{\prime}, \lambda \lambda}=2 \operatorname{Re} M^{(+)}\left(\varepsilon_{\lambda}-\varepsilon_{\lambda^{\prime}}\right) \phi_{\lambda \lambda^{\prime}}
\end{gathered}
$$

where $\phi_{\lambda \lambda^{\prime}}=\eta_{n} \phi_{n \lambda}^{2} \phi_{n \lambda^{\prime}}^{2}$, and for the interband coherences we neglect the pure dephasing and use $\gamma_{\lambda \lambda}^{(N)}=(1 / 2)\left(K_{\lambda \lambda, \lambda \lambda}+\right.$ $K_{\lambda^{\prime} \lambda^{\prime}, \lambda^{\prime} \lambda^{\prime}}$. The parameter $\eta=L^{-1}$ vanishes for the limit $L \rightarrow \infty$. This is physically justified since the relaxation in infinite systems reflects a coherent act over the whole infinite set of molecules with the probability vanishing for such an event. However, realistic systems with moderate disorder are characterized by some coherence length $\kappa$, where the realistic wave functions are delocalized. We thus use $\eta=\kappa^{-1}$ and leave this number as a fitting parameter.

The approach presented here bears considerable advances compared to the effective model of coupled oscillators used in the analysis of $2 \mathrm{D}$ electronic spectra of $\mathrm{C} 8 \mathrm{O} 3$. $^{11,12}$ On the one hand, the new treatment uses the Green's function solution of the nonlinear exciton equations (NEE) and the exciton scattering matrix for the description of the double-exciton resonances. On the other hand, the bitubular architecture of C8S3 and its energyspace coordinates are taken into account explicitly in this simulation, which yields solutions for 2D correlation and relaxation spectra in the limit of periodic boundary conditions.

Results of Homogeneous Limit Calculations. Despite the limitations inherent to an infinite model, the linear spectra are well reproduced by the simulation result, as shown in Figure 2c. The stick spectrum of the infinite lattice features two longitudinal transitions peaking at 16508 and $16904 \mathrm{~cm}^{-1}$, and two transversal transitions located at $16920 \mathrm{~cm}^{-1}$ (weak) and $17236 \mathrm{~cm}^{-1}$ (dominant). Each of the transversal transitions stems from two energy-degenerate contributions with orthogonal transition dipole moments. Note that in the homogeneous limit, the two longitudinal transitions can be spatially assigned the inner and outer wall, respectively, while the excitonic states giving rise to the two transversal transitions are spatially shared between both of the walls. By introducing a system-bath coupling strength of $280 \mathrm{~cm}^{-1}$ (time scale $50 \mathrm{fs}$ ), the experimental LA spectrum is well reproduced by the simulation results, apart from a slight energy shift of band I and an overestimated spectral resolution of band III.

As the essential features of the 2D spectra are similar at both excitation polarizations, for the present context of experiment and theory, we resort to a comparison with spatially averaged signals (i.e., to simulations of the isotropic nonlinear response). As illustrated in Figure 10, despite a systematic overestimation of band II signal strength, the key spectral properties of the experimental 2D signals can be reasonably reproduced in the homogeneous limit. We assign the systematic overestimation of band II intensity to the limitations of the present model, in particular, on the one hand, input parameters like the reduced number of sites, and, on the other hand, approximations invoked in the calculation itself (like the inclusion of zero-momentum exciton states only). Nevertheless, in the correlation spectra, we recover the asymmetry of the two triangular 2D signal parts. Though the diagonal signal of band III is virtually missing on the normalized scale, its cross-correlation with bands II and I is readily perceived. Similar is true for the intensity rearrangements in the sequence of 2D relaxation spectra. As can be seen by comparing the experimental and simulated spectra for $t_{2}=$ $1000 \mathrm{fs}$, the agreement thereby improves with increasing time delays, except for the cross-peaks in the $\omega_{3}>\left|\omega_{1}\right|$ signal part, which are too intense in the simulation. We attribute the relative overestimation of energy uphill transfer rates to mainly originate from experimentally observed excited-state population decay by fluorescence from band I, which is not included in the model.

In essence, the calculations do show best agreement with experiment if the low energy regions of the 2D spectra are compared (i.e., bands I and II). This is remarkable insofar, as due to the symmetry of the perfectly ordered structure, in the homogeneous limit, as our model involves only four states with a nonzero transition moment. Thus, in the experiment, the spectral properties of the corresponding bands are presumably 

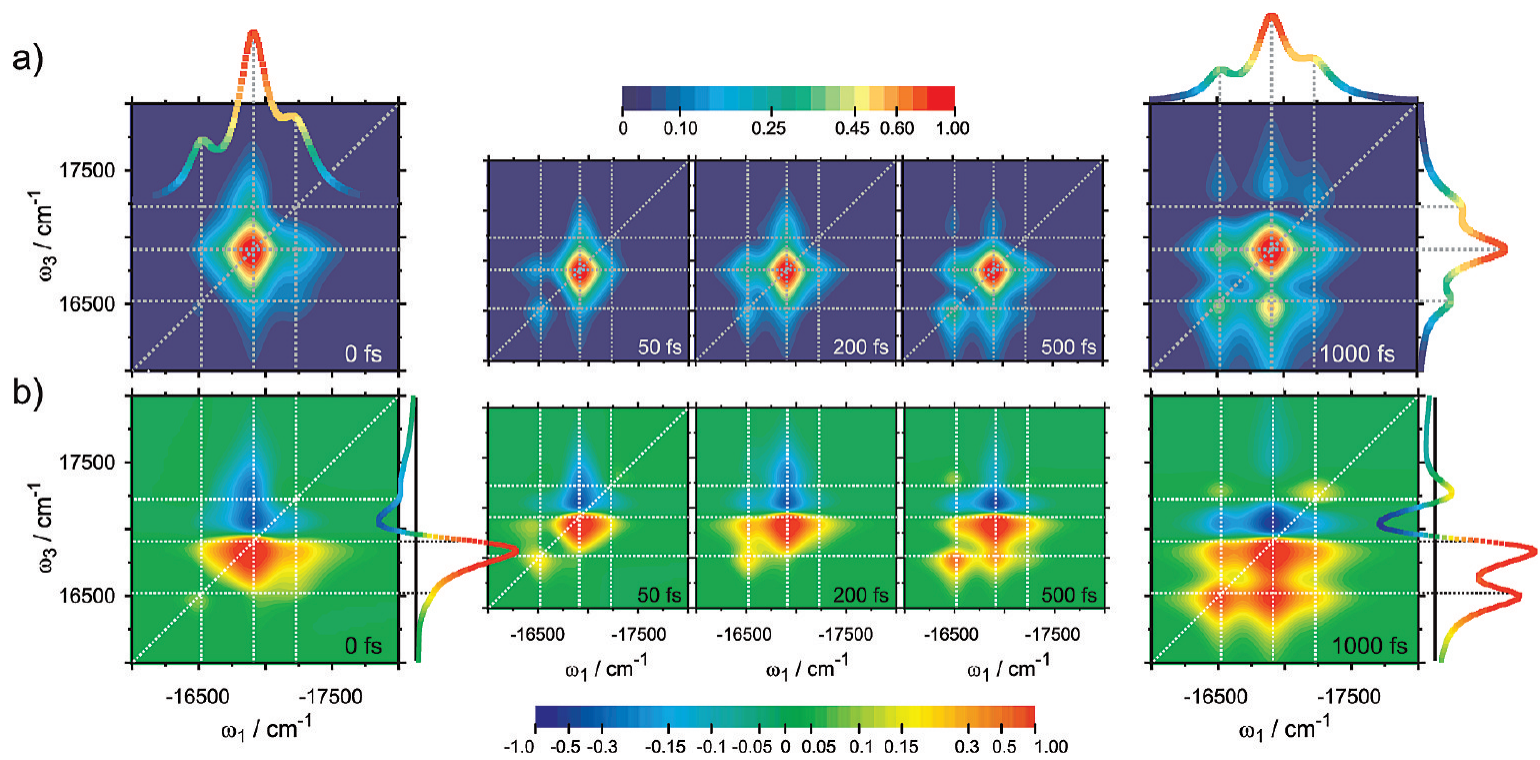

Figure 10. (a) Amplitude and (b) absorptive parts of simulated (spatially averaged) 2D electronic spectra recorded for $t_{2}$-delays of $0,50,200,500$, and $1000 \mathrm{fs}$, respectively. The corresponding (normalized) linear absorption spectra are shown for comparison in the first and last 2D spectrum in panel (a), whereas the curves in the side panels of the first and the last column in panel (b) show the 2D signals integrated along $\omega_{1}$ and are equivalent to frequency resolved pump-probe spectra. Dotted guidelines mark the absorption maxima. All spectra are normalized to their respective absolute maximum value.

determined by a relatively small number of electronic levels only. On the other hand, the limitations of the few-level model become increasingly apparent at higher frequencies. The ongoing elongation of the cross-peak and the diagonal contour of band II, both stretching far into the high energy region of $\left|\omega_{1}\right|$, are not fully reconstructed. Apart from deviations that arise from the assumption of purely homogeneous lineshapes, this finding reflects a growing density of excitonic states, accompanied by a decrease of the average oscillator strength, as discussed above.

\section{Conclusions}

The present sample is only one out of the available tubular supramolecules. Nevertheless, we expect our key conclusions to be general, and to hold also for structures that are either spatially even more complex (like interwoven tubules ${ }^{12,14,15}$ ), or whose detailed microscopic structure is tuned toward new morphologies by the admixture of surfactants. ${ }^{15,41}$ In particular, a simple translation of spectral (energetic) band positions into spatial properties will generally fail in these huge systems $\left(\approx 10^{4}\right.$ chromophores as compared to, e.g., 7 chromophores in the intensively studied Fenna-Matthews-Olson protein ${ }^{23,42}$ ), due to the delocalization of excitons induced by both intra- and interwall couplings. It is the consideration of all electronic interactions, in the framework of an excitonic Hamiltonian basis, which provides an appropriate description of the experimental data.

It is essential to note that the behavior described in Section III.3, even though with variably stringent contrasts, is observed for a wide range of disorder parameters. We thus underline the implications for not only the spatio-energetic assignment of absorption bands, but also for the perception of the exciton relaxation process. Because of intra- and interwall electronic interactions, all bands share at least a part of their wave function on the inner cylindrical assembly. As band I is dominated by (site-) localized transitions located on the inner tubule, the energy downhill motion of relaxing excitons is associated with an overall spatial transfer toward the inner wall and, simultaneously, a localization on a decreasing number of molecular sites. Even for quite large tubular segments, the lower energy parts of the absorption spectrum are determined by a handful of states only. Thus, the essential spectral signatures of exciton motion can be fitted either into phenomenological schemes of effective levels, ${ }^{11-13,15}$ or, as demonstrated in the present contribution, recovered in the infinite limit of a microscopic model. Our finite model results, in turn, explain why a congruence of experimental data and few-level simulations becomes increasingly difficult to achieve for the high energy region of the density-of-states. ${ }^{12,15}$ The higher the exciton frequencies, the more the consequences of molecular disorder come into play. Thereby, as also evidenced in the experimental 2D spectra, disorder affects transversal transitions (with typically high transition energies) to a greater extent than longitudinal ones (which dominate the red side of the spectrum).

The picture we draw can be readily connected to the recently reported correlated fluctuation of excitonic bands in a related tubular aggregate. ${ }^{14}$ Since we find all exciton wave functions to be at least partly located on one and the same wall, and to consequently overlap in space (i.e., to involve common molecular chromophores), also their fluctuations share a certain degree of correlation. Notably, the effect does not necessarily implicate correlated fluctuations of individual molecular sites. We further note that, even in the presence of disorder, only a relatively small number of states governs the aggregate's spectroscopic properties. Combined with the notion that fluctuation amplitudes become exchange-narrowed for delocalized (excitonic) states, our theoretical considerations are thus in line with experimentally observed interband coherences, ${ }^{13,15}$ as a consequence of a slowly dephasing, small ensemble of excitons. In a broader context, our results indicate that for large excitonic systems, interband coherences result from the physics that governs the evolution of highly delocalized electronic states, and do not necessitate a surrounding protein matrix like in small natural photosynthetic complexes. ${ }^{43}$

This contribution provides a guideline of how exciton motion in supramolecular systems can be studied, despite the limits currently set for connecting experimental and theoretical state- 
of-the-art methodologies. Though we report on an artificial light harvester that self-assembles in aqueous solution, it is inspiring to think about likely functional analogies to tubular complexes occurring in nature, ${ }^{6,44}$ which, similarly, do not require any structural templates. A forthcoming work that compares experimental results from single- as well as double-quantum 2D electronic spectroscopy ${ }^{45,46}$ against refined calculations is in progress.

Acknowledgment. J. S. and A. N. acknowledge funding by the Austrian Science Foundation FWF (AF 01618-NO2 and $P$ 22331) and partial support within the doctoral scholarship programme (DOC and DOC-fFORTE) of the Austrian Academy of Sciences. J. H. acknowledges the FWF for financial support by a Lise-Meitner scholarship. S. M. and D. A. acknowledge support by the National Science Foundation (CHE 0745892). The authors gratefully acknowledge stimulating discussions with Hans von Berlepsch (FU Berlin), who also provided the cryoTEM image shown in Figure 1a.

\section{References and Notes}

(1) Light-harvesting J-Aggregates; Knoester, J., Ed.;Special Issue of the Int. J. Photoenergy; Hindawi: New York, 2006.

(2) Kirstein, S.; Dähne, S. Int. J. Photoenergy 2006, 2006, 1-21.

(3) 3,3'-bis(3-sulfopropyl)-5,5',6,6'-tetrachloro-1,1'-dioctylbenzimidacarbocyanine.

(4) Didraga, C.; Pugžlys, A.; Hania, P. R.; von Berlepsch, H.; Duppen, K.; Knoester, J. J. Phys. Chem. B 2004, 108, 14976-14985.

(5) Prokhorenko, V. I.; Steensgaard, D. B.; Holzwarth, A. R. Biophys. J. 2003, 85, 3173-3186.

(6) Ganapathy, S.; Oostergetel, G. T.; Wawrzyniak, P. K.; Reus, M.; Chew, A. G. M.; Buda, F.; Boekema, E. J.; Bryant, D. A.; Holzwarth, A. R.; de Groot, H. J. M. Proc. Natl. Acad. Sci. U.S.A. 2009, 109, 8525-8530.

(7) Davydov, A. S. Theory of Molecular Excitons; Plenum Press: New York, 1971.

(8) Didraga, K.; Klugkist, J. A.; Knoester, J. J. Phys. Chem. B 2002, 106, 11474-11486.

(9) Collini, E.; Scholes, G. D. J. Phys. Chem. A 2009, 113, 4223.

(10) 5,5',6,6'-tetrachloro-1,1'-dioctyl-3,3'-di-(3-carboxypropyl)-benzimidacarbocyanine.

(11) Milota, F.; Sperling, J.; Nemeth, A.; Abramavicius, D.; Mukamel, S.; Kauffmann, H. F. J. Chem. Phys. 2009, 131, 054510.

(12) Nemeth, A.; Milota, F.; Sperling, J.; Abramavicius, D.; Mukamel, S.; Kauffmann, H. F. Chem. Phys. Lett. 2009, 469, 130-134.

(13) Womick, J. M.; Miller, S. A.; Moran, A. M. J. Phys. Chem. A 2009, 113, 6587-6598.

(14) Womick, J. M.; Miller, S. A.; Moran, A. M. J. Phys. Chem. B 2009, 113, 6630-6639.

(15) Milota, F.; Sperling, J.; Nemeth, A.; Kauffmann, H. F. Chem. Phys. 2009, 357, 45-53.

(16) Didraga, C.; Knoester, J. J. Lum. 2004, 110, 239.
(17) Didraga, C.; Knoester, J. J. Chem. Phys. 2004, 121, 10687-10698.

(18) Pugžlys, A.; Augulis, R.; van Loosdrecht, P. H. M.; Didraga, C.; Malyshev, V. A.; Knoester, J. J. Phys. Chem. B 2006, 110, 20268.

(19) Tauber, M. J.; Mathies, R. A.; Chen, X.; Bradforth, S. E. Rev. Sci. Instrum. 2003, 74, 4958-4960.

(20) Jonas, D. M. Annu. Rev. Phys. Chem. 2003, 54, 425-463.

(21) Cho, M. Chem. Rev. 2008, 108, 1331-1418.

(22) Abramavicius, D.; Palmieri, B.; Voronine, D. V.; Sanda, F.; Mukamel, S. Chem. Rev. 2009, 109, 2350-2408.

(23) Brixner, T.; Stenger, J.; Vaswani, H. M.; Cho, M.; Blankenship,

R. E.; Fleming, G. R. Nature 2005, 434, 625.

(24) Cho, M.; Vaswani, H. M.; Brixner, T.; Stenger, J.; Fleming, G. R. J. Phys.Chem. B 2005, 109, 10542-10556.

(25) Milota, F.; Sperling, J.; Nemeth, A.; Mancal, T.; Kauffmann, H. F. Acc. Chem. Res. 2009, 42, 1364.

(26) Piel, J.; Riedle, E.; Gundlach, L.; Ernstorfer, R.; Eichberger, R. Opt. Lett. 2006, 31, 1289-1291.

(27) Baum, P.; Breuer, M.; Riedle, E.; Steinmeyer, G. Opt. Lett. 2006, $31,2220-2222$.

(28) Baum, P.; Lochbrunner, S.; Riedle, E. Opt. Lett. 2004, 29, 210212.

(29) Cowan, M. L.; Ogilvie, J. P.; Miller, R. J. D. Chem. Phys. Lett. 2004, 386, 184-189.

(30) Brixner, T.; Mančal, T.; Stiopkin, I. V.; Fleming, G. R. J. Chem. Phys. 2004, 121, 4221-4236.

(31) Hochstrasser, R. M. Nature 2005, 434, 570.

(32) Stiopkin, I.; Brixner, T.; Yang, M.; Fleming, G. R. J. Phys. Chem. B 2006, 110, 20032-20037.

(33) Ginsberg, N. S.; Cheng, Y.-C.; Fleming, G. R. Acc. Chem. Res. 2009, 42, 1352.

(34) Cheng, Y.-C.; Fleming, G. R. Annu. Rev. Phys. Chem. 2009, 60, 241.

(35) Read, E. L.; Lee, H.; Fleming, G. R. Photosynth. Res. 2009, 101, 233.

(36) Mukamel, S.; Abramavicius, D. Chem. Rev. 2004, 104, 2073.

(37) Mukamel, S. Principles of Nonlinear Optical Spectroscopy; Oxford University Press: New York, 1995.

(38) Chernyak, V.; Zhang, W. M.; Mukamel, S. J. Chem. Phys. 1998, 109, 9587.

(39) Spano, F. C.; Mukamel, S. J. Chem. Phys. 1991, 95, 7526.

(40) Chernyak, V.; Mukamel, S. J. Chem. Phys. 1996, 105, 4565.

(41) von Berlepsch, H.; Kirstein, S.; Bttcher, C. Langmuir 2002, 18, 7699-7705.

(42) Read, E. L.; Schlau-Cohen, G. S.; Engel, G. S.; Wen, J.; Blankenship, R. E.; Fleming, G. R. Biophys. J. 2008, 95, 847-856.

(43) Engel, G. S.; Calhoun, T. R.; Read, E. L.; Ahn, T. K.; Mančal, T.; Cheng, Y. C.; Blankenship, R. E.; Fleming, G. R. Nature 2007, 446, 782786.

(44) Fetisova, Z. G.; Freiberg, A. M.; Timpmann, K. E. Nature 1988, 334, 633-634.

(45) Nemeth, A.; Sperling, J.; Hauer, J.; Kauffmann, H. F.; Milota, F. Opt. Lett. 2009, 34, 3301-3303.

(46) Nemeth, A.; Milota, F.; Mančal, T.; Pullerits, T.; Sperling, J.; Hauer, J.; Kauffmann, H. F.; Christensson, N. J. Chem. Phys. 2010, 133 (6), in press.

JP102173N 